\title{
Computational intelligence approaches for student/tutor modelling: a review
}

\begin{abstract}
The intelligent tutoring system (ITS) is an educational software system that provides personalized and adaptive tutoring to students based on their needs, profiles and preferences. The tutor model and student model are two dependent components of any ITS system. The goal of any ITS system is to help the students to achieve maximum learning gain and improve their engagements to the systems by capturing the student's interests through the system's adaptive behavior. In other words an ITS system is always developed with the aim of providing an immediate and efficient solution to student's learning problems. In recent years a lot of work has been devoted to improving student and tutor models in order enhance the teaching and learning activities within the ITS systems. The aim of this paper is to investigate the most recent state of art in the development of these two vital components of the intelligent tutoring systems.
\end{abstract}

Keyword: Tutor model; Student model; Computational techniques; Intelligent tutoring systems 